\title{
Linear in temperature correction to the Casimir force
}

\author{
V.B. Svetovoy* and M.V. Lokhanin \\ Department of Physics, Yaroslavl State University, \\ Sovetskaya 14, Yaroslavl 150000, Russia
}

()

\begin{abstract}
We discuss the temperature correction to the Casimir force between nonideal metallic bodies which caused disagreement in the literature. A general method to find the troubling term is proposed that does not require a direct reference to the Lifshitz formula. The linear in temperature correction is shown to survive for nonideal metals. It is important for small separations between bodies tested in the recent experiments.
\end{abstract}

12.20.Ds, 03.70.+k

The Casimir force [1] has been measured with high precision in recent experiments [2 5 ] between metallized sphere and plate. Also there are plans [6.7] to look for very weak hypothetical forces where the Casimir force is the main background. All this makes the precise evaluation of the Casimir force an important problem. Here we will discuss a particular problem concerning the temperature dependence of the force between macroscopic bodies made of real metals. Discussion of this problem in the literature revealed disagreement between different authors and at the moment there are three different results.

For perfect conductors the temperature correction has been found many years ago 8 10 and it is small for small separations between bodies $a \ll c \hbar / k T$ or equivalently for low temperature. For a sphere above a plate the leading term behaves as $\left(T / T_{e f f}\right)^{3}$, where $k T_{\text {eff }}=\hbar c / 2 a$. This result follows from a general expression for the Casimir force given by Lifshitz [11,12] modified for the case of sphere-plate geometry with the proximity force theorem (PFT) [13]:

$$
F(a)=-\frac{k T R}{4 a^{2}} \sum_{n=0}^{\infty} \int_{x_{n}}^{\infty} d x x \ln \left[\left(1-G_{1} e^{-x}\right)\left(1-G_{2} e^{-x}\right)\right],
$$

where $R$ is the sphere radius,

$$
\begin{gathered}
G_{1}=\left(\frac{x-s}{x+s}\right)^{2}, \quad G_{2}=\left(\frac{\varepsilon\left(i \zeta_{n}\right) x-s}{\varepsilon\left(i \zeta_{n}\right) x+s}\right)^{2}, \\
s=\sqrt{x_{n}^{2}\left(\varepsilon\left(i \zeta_{n}\right)-1\right)+x^{2}}, \quad x_{n}=\frac{2 \zeta_{n} a}{c}, \quad \zeta_{n}=\frac{2 \pi n k T}{\hbar}
\end{gathered}
$$

$\varepsilon\left(i \zeta_{n}\right)$ is the dielectric function of the used material at imaginary frequencies. The prime over the sum sign indicates that the first term $n=0$ has to be taken with the coefficient $1 / 2$. Throughout this paper we will work with the force $F(a)$ between sphere and plate. The force for the plate-plate configuration can be restored as $F^{\prime}(a) / 2 \pi R$.

For small temperature the sum in (1) can be replaced by the integral and the resulting force does not depend on the temperature at all. This limit has been considered [14,15] to calculate the force in the conditions of experiments. In general, the replacement is true with the precision $\sim T / T_{\text {eff }}$. For the atomic force microscope (AFM) experiments [3, 1 the smallest separation was $0.1 \mu \mathrm{m}$ and the replacement error can be as large as $3 \%$ at $T=300^{\circ} \mathrm{K}$. It exceeds the experimental errors $\sim 1 \%$ and, therefore, the finite temperature effect has to be taken into account. We define the temperature correction $\Delta_{T} F$ as the difference between forces written as the sum over $n$ and as the integral instead of this sum.

The physical discussion of the first term in the sum (11) raised heated debate in the literature. It has a long history. For ideal metals the temperature correction found from the Lifshitz formula [16] did not agree with that found with different methods [8,9]. Schwinger et al. [10] clearly demonstrated that when the static limit was correctly taken in the Lifshitz theory the discrepancy disappeared. The formulated condition is known as the Schwinger prescription: $\varepsilon$ should approach infinity before the frequency is allowed to go to zero.

Different results have been found for the $n=0$ term in the recent works where the Casimir force was calculated between nonideal metals. All the results one can parametrize [17] with the following relation

*E-mail: svetovoy@nordnet.ru 


$$
F_{n=0}(a)=\alpha \frac{k T R}{4 a^{2}} \zeta(3),
$$

where $\zeta(m)$ is the zeta-function and $\alpha$ is the parameter. In our paper $[18] \alpha=1$ was found using the Drude model for $\varepsilon(i \zeta)$ and the Schwinger prescription. Doubt in applicability of the prescription for real metals was expressed in [19] where straightforward calculation gave $\alpha=1 / 2$. Using the plasma model for the metal dielectric function, $\alpha=1-2 c / a \omega_{p}$ was found [20] (see also [21]), where $\omega_{p}$ is the plasma frequency. Physical arguments based on the finite size of plates in real experiments have led Lamoreaux to the result $\alpha=1$ [17]. There is no disagreement for the other terms in the sum (11), so different $\alpha$ are responsible for different temperature corrections. In this letter we will try to clarify the problem.

The $n=0$ term (3) gives the classical contribution to the force ( $\hbar$-independent). At large separations between bodies $\left(T_{\text {eff }} \ll T\right)$ this is the only term in the sum (11) which survives. All the other terms are exponentially suppressed. In this sense one can say that (3) describes the contribution of the long wavelength or low frequencies fluctuations (the static limit). Below we demonstrate that the value of $\alpha$ can be found for real metals from very general analysis without direct reference to the Lifshitz formula (11) if one knows its value for ideal metals. It is important because the static limit calculated from (1i) is controversial: it gives different values for $\alpha$ if the metal is described by the plasma or the Drude models and there is no way to reconcile the values in the limit of zero relaxation frequency.

The force in the classical limit (3) with an arbitrary parameter $\alpha$ can be written using only the dimensional analysis. More exactly one can reproduce the force between two plates and then apply PFT. The value of $\alpha$ can depend on the material parameters which define the metal dielectric function $\varepsilon(i \zeta)$ at low frequencies. In this limit $\varepsilon(i \zeta)$ depends on the only one parameter which is the metal conductivity $\sigma$ or equivalently the resistivity $\rho=1 / \sigma$. The dimensionless parameter $\alpha$ can be a function of the only one dimensionless variable

$$
\alpha=\alpha\left(\frac{c \rho \varepsilon_{0}}{a}\right),
$$

where $\varepsilon_{0}$ is the free space permittivity. For ideal metals $\rho \rightarrow 0$ and $\alpha(0)=1$ as was established many years ago [8 10$]$. Let us estimate the length scale $L=c \rho \varepsilon_{0}$ for real good metals. Using the typical resistivity $\rho \leq 10 \mu \Omega \cdot \mathrm{cm}$ one finds $L \leq 3 \AA$. Of course, one can expand the function $\alpha(L / a)$ in the powers of $L / a$ but there is no too much sense in this. The reason is that $L$ is a microscopic scale. Really, it is closely connected with the screening length $L_{s} \sim v_{F} / \omega_{p}$ of degenerated electron gas, where $v_{F}$ is the Fermi velocity of electrons. If $\delta=c / \omega_{p}$ is the field penetration depth and $l$ is the mean free path for electrons, then $L \sim L_{s}(\delta / l)$. For good metals $\delta \sim l$ and $L$ is the same order as $L_{s}$. Taking into account such a short scale will be excessive for the macroscopic Casimir force. Therefore, we can conclude that the force in the static limit is the same as for ideal metals, i.e. $\alpha=1$. Intuitively it seems quite obvious. In the static limit there is no difference between metals because the free charges manage to follow the slowly varying field. That is why the boundary conditions in this case do not include any characteristic of a particular metal. This conclusion means that if we try to calculate the force using the Lifshitz formula (1), the Schwinger prescription has to be applied to the $n=0$ term independently if it is a perfect or real metal. Lamoreaux [17] arrived at the same conclusion from quite different physical consideration.

In ref. [19] fair calculation of the $n=0$ term in (11) gave $\alpha=1 / 2$. This value does not depend on any material parameter and, therefore, should be the same as for the ideal metal. However, we know that for a perfect conductor $\alpha=1$ due to the Schwinger prescription. So the disagreement has the same nature as in the old story described above.

In ref. [20] the plasma model was used for the metal dielectric function

$$
\varepsilon(i \zeta)=1+\frac{\omega_{p}^{2}}{\zeta^{2}} .
$$

This model gives good description of a metal in the infrared range but cannot be used at low frequencies where $\varepsilon(i \zeta)=1+\left(\varepsilon_{0} \rho \zeta\right)^{-1}$. Therefore, it will be good everywhere except of the $n=0$ term for which one has to use the Schwinger prescription. It is no wonder then that in the static limit a significant correction to $\alpha=1$ has been found [20]: $\alpha=1-2 c / a \omega_{p}$. That is because in the plasma model the length scale $L=c / \omega_{p}$ (penetration depth) becomes macroscopic $L>150 \AA$ and expansion in $L / a$ has sense.

The plasma model cannot be used at low frequencies even in principle. The only way to get $\varepsilon(i \zeta)$ is the dispersion relation

$$
\varepsilon(i \zeta)=1+\frac{2}{\pi} \int_{0}^{\infty} d \omega \frac{\omega \varepsilon^{\prime \prime}(\omega)}{\omega^{2}+\zeta^{2}},
$$


where $\varepsilon^{\prime \prime}(\omega)$ is the imaginary part of the dielectric function at real frequencies. In the plasma model there is no dissipation and $\varepsilon^{\prime \prime}(\omega)=0$. Eq. (5) one can derive from (6) only calculating the integral with some small but finite relaxation frequency $\omega_{\tau}$. For finite dissipation always there is such a frequency for which $\varepsilon(i \zeta)=1+\left(\varepsilon_{0} \rho \zeta\right)^{-1}$ with an arbitrary small $\rho$.

The authors 20] claim that some identical transformation of the Lifshitz formula removes the controversy in the force for the plasma and Drude models without the use of Schwinger prescription. This statement is definitely wrong because any identical transformation cannot change the result. Below it will be demonstrated explicitly.

Let us discuss now the temperature correction to the Casimir force. The sum for $n>0$ in (11) as a function of temperature contains a piece linear in $T$ which exactly cancels for ideal metals the $n=0$ term giving in the small temperature limit $T / T_{\text {eff }}<1$ the well known result [9]

$$
F_{T}(a)=F_{0}(a)\left[1+\frac{45 \zeta(3)}{\pi^{3}}\left(\frac{T}{T_{e f f}}\right)^{3}-\left(\frac{T}{T_{e f f}}\right)^{4}\right]
$$

where $F_{0}(a)=\pi^{3} \hbar c R /\left(360 a^{3}\right)$ is the bare Casimir force between sphere and plate.

If we are using the dielectric function of a real metal, the cancellation of the first term in (1) is incomplete and the linear in $T$ contribution survives. That was noted first in [18], where eq. (11) with $\alpha=1$ was used for numerical evaluation of the Casimir force. It was found that for the AFM experiments [3] 4 ] the temperature correction at the smallest separation is $4 p N$ against the experimental errors $1 p N$. We can give now an explicit expression for the correction.

In ref. [20] the authors did not use the Schwinger prescription in the $n=0$ term and took the plasma model for $\varepsilon(i \zeta)$. For this case they convincingly demonstrated that there was no the linear in $T$ correction and the leading correction is only $\left(T / T_{e f f}\right)^{3}$. As was shown above the prescription must be applied to the $n=0$ term so the only difference in evaluation of the sum comes from this term. This difference is just the correction we are looking for

$$
\Delta_{T} F=\frac{k T R}{4 a^{2}}\left\{\zeta(3)+\frac{1}{2} \int_{0}^{\infty} d x x \ln \left[\left(1-G_{1} e^{-x}\right)\left(1-G_{2} e^{-x}\right)\right]\right\}+O\left(\left(T / T_{e f f}\right)^{3}\right) .
$$

The integral here can be interpreted as the linear in $T$ term contained in the sum (1) for $n>0$ and, of course, it can depend on the material parameters because the summation is going over nonzero frequencies $\zeta_{n}$. On the other hand, since this integral appeared as the $n=0$ term in (11), we should take the functions $G_{1,2}$ at $x_{n}=0$. In this limit $G_{2}=1$ but $G_{1} \neq 1$. Using then the relation $\int_{0}^{\infty} d x x \ln \left(1-e^{-x}\right)=-\zeta(3)$ one finds the final expression for the correction linear in $T$ :

$$
\Delta_{T} F=\frac{k T R}{8 a^{2}}\left[\zeta(3)+\int_{0}^{\infty} d x x \ln \left(1-G_{1} e^{-x}\right)\right]+O\left(\left(T / T_{e f f}\right)^{3}\right)
$$

where

$$
G_{1}=\left(\frac{x-\sqrt{x^{2}+\beta^{-2}}}{x+\sqrt{x^{2}+\beta^{-2}}}\right)^{2}, \quad \beta=\frac{c}{2 a \omega_{p}} .
$$

Let us stress that (9) is true only for the plasma model. When $\omega_{p} \rightarrow \infty$ the correction disappears as it should be. Expansion in powers of $\beta$ gives

$$
\Delta_{T} F=\frac{k T R}{8 a^{2}} \zeta(3) \cdot 8 \beta\left(1-3 \beta+O\left(\beta^{2}\right)\right)+O\left(\left(T / T_{e f f}\right)^{3}\right) .
$$

For $\omega_{p}=2 \cdot 10^{16} \mathrm{~s}^{-1}, a=0.1 \mu \mathrm{m}$ and $R=100 \mu \mathrm{m}$ as in the AFM experiments [3, 4 , one gets $\Delta_{T} F \approx 2.5 p N$ using (9) or calculating directly with the help of (11) and $2.9 \mathrm{pN}$ using (10). In the condition of the Lamoreaux experiment [2] $\left(\omega_{p}=1.4 \cdot 10^{16} \mathrm{~s}^{-1}, a=0.6 \mu \mathrm{m}\right.$ and $\left.R=12.5 \mathrm{~cm}\right)$ the linear in $T$ part of the correction $\Delta_{T} F \approx 29 \mathrm{pN}$ is within the experimental errors. This conclusion is in contrast with that of Boström and Sernelius [19] due to obvious reason. If $\alpha=1 / 2$ as in ref. [19], the $n=0$ term is not canceled by the sum of those at $n>0$ and there is no an additional suppression $\sim \beta$ as in (10).

The correction increases further if we will use the Drude dielectric function 


$$
\varepsilon(i \zeta)=1+\frac{\omega_{p}^{2}}{\zeta\left(\zeta+\omega_{\tau}\right)} .
$$

In this case it has to be evaluated numerically using (1) and subtracting the same formula with the integral instead of the sum. The relaxation frequency $\omega_{\tau}$ influences mostly on the integral since it changes low frequency behavior of the integrand. For typical value $\omega_{\tau}=5 \cdot 10^{13} \mathrm{~s}^{-1}$ we found $\Delta_{T} F \approx 4.0 \mathrm{pN}$ in condition of the AFM experiments [3., This value coincide with that reported before [18]. In the experiment [5] the smallest separation was $a=63 \mathrm{~nm}$ and the absolute value of the correction is larger $\Delta_{T} F \approx 10 p N$ [22] though its relative value decreases. It has to be compared with the experimental error $3.5 \mathrm{pN}$. Note that this correction make the agreement between theory and experiment better [22].

In ref. 20 the authors claim that the Drude model has a principal drawback which is discontinuity of $G_{1}$ at $x=0$ for the $n=0$ term. Namely, $G_{1}=1$ at $x=0$ but $G_{1}=0$ at $x \neq 0$ in the limit $x_{0} \rightarrow 0$. In this connection we would like to stress that the finite discontinuity in one point has no effect on the integral in (11). As was explained above the problem with the $n=0$ term has the physical nature. One has to accurately takes into account the static limit for which $\varepsilon \rightarrow \infty$ must be taken before allowing $\zeta \rightarrow 0$ for both real and ideal metals.

The problem with the $n=0$ term was declared [20] to be connected with the discontinuity of $G_{1}$. This has led the authors 20] to the wrong conclusion that the problem can be solved by the mathematical methods. They transformed eq. (11) with the Poisson formula and represented it as the Fourier transformation

$$
F(a)=-\frac{k T R}{2 a^{2}} \sum_{m=0}^{\infty} \int_{0}^{\infty} d t \cos (2 \pi m t) \varphi(\tau t)
$$

where

$$
\varphi(z)=\int_{z}^{\infty} d x x \ln \left[\left(1-G_{1} e^{-x}\right)\left(1-G_{2} e^{-x}\right)\right], \quad \tau=2 \pi \frac{T}{T_{e f f}} .
$$

It was stated that in this form the Lifshitz formula had no problem for the Drude model. However, the Poisson formula is just an identical transformation for the series and it cannot give any new result. As we know without the Schwinger prescription it follows from (11) that $\alpha=1 / 2$ 19]. The same value one can get from (12). It was demonstrated numerically 23 and easy to do analytically.

To see it explicitly, let us consider the static limit $\tau \gg 1$. Calculating the sum in (12) for finite but large $M \gg \tau$ one finds

$$
F(a)=-\frac{k T R}{4 a^{2} \tau} \int_{0}^{\infty} d z \frac{\sin \pi(2 M+1) z / \tau}{\sin \pi z / \tau} \varphi(z) .
$$

The integral is saturated at $\pi z / \tau \sim 1 /(2 M+1) \ll 1$ and we can replace $\sin \pi z / \tau \approx \pi z / \tau$. Then (13) can be represented as

$$
F(a)=-\frac{k T R}{4 \pi a^{2}} \int_{0}^{\infty} d y \frac{\sin y}{y} \varphi\left(\frac{\tau y}{\pi(2 M+1)}\right) .
$$

We get the expected result from (14) taking the limit $M \rightarrow \infty$ :

$$
F(a)=-\frac{k T R}{8 a^{2}} \int_{0}^{\infty} d x x \ln \left[\left(1-G_{1} e^{-x}\right)\left(1-G_{2} e^{-x}\right)\right] \quad \text { for } \tau \gg 1 .
$$

It coincide with the $n=0$ term in (11) as it should be. Therefore, using the Drude model we will get $\alpha=1 / 2$ from (12) so as from the original Lifshitz formula (1).

In conclusion, we have considered the linear in temperature correction to the Casimir force at low temperatures or equivalently at small separations. Special care has to be taken to get the contribution of the fluctuations in the static limit $(n=0$ term). This contribution is canceled for ideal mirrors but cancellation is incomplete for real metals. A general method based on the dimensional analysis has been proposed to calculate the troubling $n=0$ term without 
direct reference to the Lifshithz formula. On this bases it was shown that the Schwinger prescription had to be applied in the static limit independently if it is real or ideal metal. The static contribution is the same for the plasma and Drude models. The temperature correction is shown to be important for the AFM experiments [3 5] at the smallest separations but is less than the experimental errors for the torsion pendulum experiment [2].

[1] H.B.G. Casimir, Koninkl. Ned. Adak. Wetenschap. Proc. 51 (1948) 793.

[2] S.K. Lamoreaux, Phys. Rev. Lett. 78 (1997) 5; 81 (1998) 5475.

[3] U. Mohideen and A. Roy, Phys. Rev. Lett. 81 (1998) 4549.

[4] A. Roy, C. -Y. Lin, and U. Mohideen, Phys. Rev. D60 (1999) 111101.

[5] B.W. Harris, F. Chen and U. Mohideen, Phys. Rev. A62 (2000) 052109.

[6] J.C. Long, H.W. Chan, and J.C. Price, Nucl. Phys. B539 (1999) 23.

[7] E. Fischbach and C. Talmadge, The Search for Non-Newtonian Gravity (AIP Press/Springer-Verlag, New York, 1999).

[8] J. Mehra, Physica 37 (1967) 145.

[9] L.S. Brown and G.J. Maclay, Phys. Rev. 184 (1969) 1272.

[10] J. Schwinger, L.L. DeRaad, Jr., and K.A. Milton, Ann. Phys. (N.Y.) 115 (1978) 1.

[11] E.M. Lifshitz, Sov. Phys. JETP 2 (1956) 73.

[12] E.M. Lifshitz and L.P. Pitaevskii, Statistical Physics, Part 2 (Pergamon Press, Oxford, 1980).

[13] J.Blocki, J. Randrup, W.J. Swiatecki, and C.F. Tsang, Ann. Phys. (N.Y.) 105 (1977) 427.

[14] S.K. Lamoreaux, Phys. Rev. A59 (1999) R3149.

[15] A. Lambrecht and S. Reynaud, Eur. Phys. J. D8 (2000) 309.

[16] I.E. Dzyaloshinskii, E.M. Lifshitz, and L.P. Pitaevskii, Sov. Phys. Usp. 4 (1961) 153.

[17] S.K. Lamoreaux, e-print quant-ph/0007029.

[18] V.B. Svetovoy and M.V. Lokhanin, e-print quant-ph/0001010; Mod. Phys. Lett. A15 (2000) 1013.

[19] M. Boström and Bo E. Sernelius, Phys. Rev. Lett. 84, 4757 (2000).

[20] M. Bordag, B. Geyer, G.L. Klimchitskaya, and V.M. Mostepanenko, Phys. Rev. Lett. 85 (2000) 503.

[21] C. Genet, A. Lambrecht, and S. Reynaud, Phys. Rev. A 62 (2000) 012110.

[22] V.B. Svetovoy and M.V. Lokhanin, Mod. Phys. Lett. A15 (2000) 1437.

[23] M. Boström, private communication. 